BULLETIN (New Series) OF THE

AMERICAN MATHEMATICAL SOCIETY

Volume 37, Number 1, Pages 45-53

S 0273-0979(99)00803-4

Article electronically published on December 21, 1999

\title{
CURRENT TENDENCIES OF MATHEMATICAL RESEARCH
}

\author{
EDWARD B. VAN VLECK
}

In an address which I had the pleasure of giving at the Atlanta meeting of the American Association for the Advancement of Science, I traced the influence of Fourier's series upon the development of mathematics. This instance is only one of many in which a central thought or problem has exerted a remarkable, sometimes even a controlling influence upon the development of mathematical thought. From early Greek times I may cite the historic problems of the duplication of the cube and the squaring of the circle. After the invention of analytic geometry it became essential to determine the tangent at a point of a given curve, and this led, as you know, to the discovery of the differential calculus, one of the most wonderful instruments ever devised for scientific thought. Almost simultaneously the problem of finding the area of a curve resulted in the construction of the definite integral, a concept even wider in scope than the differential coefficient. Still earlier, and near the beginning of the mathematical renaissance, the problem afforded by the equation of the $n$th degree was brilliantly solved for equations of the third and fourth degrees, and the first creative impulse subsequent to Greek times was thereby given to mathematics. In the last half of the nineteenth century this same problem eventuated in the theory of groups which has become the foundation of mathematical classification. $^{1}$

Examples such as these prompt us to ask: What are the central problems in the mathematical research of to-day? Each mathematical period has its own characteristics. The discovery of the calculus was followed by a notable period which I shall term the differential and gravitational epoch of mathematics, in which the consequences of the new calculus absorbed the attention, while at the same time it was developed as the handmaid of astronomy and physical science. The names of Euler on the one side, of Lagrange and Laplace on the other, serve sufficiently to specify the era and its two-fold aspects. For many reasons the last quarter of the nineteenth century might be termed the group period, from which we have only in part emerged. I need not tell you how necessary it became to explore geometry and analysis under the guidance of the group concept. Yet there are reservations which must be made in designating this quarter century as the group period. Likewise it is necessary to qualify any answer to the difficult question: What is the dominant

2000 Mathematics Subject Classification. Primary 01A55, 01A60.

Reprinted from Bull. Amer. Math. Soc. 23 (1916), 1-13.

This address was read at the Quarter Centennial of the University of Chicago before a conference of the mathematical, physical, and astronomical departments.

${ }^{1}$ Many kinds of geometries and number fields have come to be distinguished. Their study and correlation are a feature of modern mathematical research which I have not found it convenient to discuss in this address. 
problem or central thought in the research of to-day, if there be one? Nevertheless, let us be so importunate as to insist upon an answer, however foolhardy.

In the field of applied mathematics probably the "problem of three bodies" can be picked out as par excellence the present-day problem. Already in the hands of Poincaré this has given a first quickening of the fossilized methods of mathematical astronomy. In pure mathematics, to which I shall confine my attention, the number of conspicuous problems is legion, but above them all there looms, I think, in manifold aspects the problem of the infinite set. Analysis, geometry, and mechanics alike have been rapidly and increasingly permeated by the point set theory of Georg Cantor, in which the central core is the problem of the infinite set. More and more do the problems in these branches require, in some form or other, upto-date knowledge of the infinite set. Consider, for example, what is called the problem of the primitive function. Given a function $F(x)$, what conditions must be imposed upon it in order that there shall exist another or "primitive function" whose derivative is the given function? Or take the alternative question: What generalization shall be made in the notion of a derivative in order that as wide a class of functions as possible shall possess a primitive? Answers to these questions must involve a careful study of the infinite set of discontinuities of a function.

The characteristic tendency in the thought of to-day which I have tried to grasp under the comprehensive term "Problem of the infinite set" is shown rather as a current beneath the surface than in any individual concrete problem. The average investigator must perforce seize upon any problem which his brains find at hand. There exist, however, certain fundamental principles which will aid him in finding a worthy one. The great mathematician Jacobi is said to have inculcated upon his students the dictum: Man muss immer umkehren. One must always seek a converse, turn a thought the other end to. It was by turning the elliptic integral inside out that Jacobi obtained his splendid theory of elliptic and theta functions. As other instances of the same principle I cite at random the implicit function theory which underlies so much modern investigation, and the perception of the existence of transcendental numbers through failure of the finite processes which produce our ordinary numbers.

Without dwelling further upon the fertility of Jacobi's dictum, I wish to coin and put beside it another obvious dictum of yet wider reach: Man muss immer generalizieren. By this I do not mean cheap generalization to $n$ dimensions or variables of that which has been already done for two or three. Not infrequently, however, generalization even to $n$ variables is a problem of importance and difficulty, inasmuch as the solution for the special case of two or three variables may possess a distinctive structure and character and hence be in no way typical. A notable instance of such difficulty is found in the generalization of the ordinary second order conditions for the maximum of a definite integral in the calculus of variations, while extraordinary obstacles are encountered in the extension of the theory of analytic functions to two or more variables. But whether or not extension to $n$ dimensions be trivial, generalization to a countably infinite number of dimensions becomes sublime! With a further extension to an uncountably infinite number of dimensions or variables it might seem that we would reach a sublimated or etherial stage of mathematical development, but such flippant characterization would give an altogether false impression of Volterra's theory of functions of lines.

Extension to a countably infinite number of variables commonly affords opportunity for the finest sagacity and insight. An excellent program for work could be 
found in extension of almost any finite theory. It is your own Professor Moore whom I have heard glowingly preach that to every finite theory there must correspond, under proper limitations, a general transcendental theory with an infinite number of variables. What is more beautiful and simple in elementary mathematics than the application of the theory of determinants to the solution of a set of $n$ linear equations in $n$ variables

$$
\begin{aligned}
& \left(1+a_{11}\right) x_{1}+a_{12} x_{2}+\cdots+a_{1 n} x_{n}=C_{1}, \\
& a_{1 n} x_{1}+a_{2 n} x_{2}+\cdots+\left(a_{n n}+1\right) x_{n}=C_{n} ;
\end{aligned}
$$

such a system as we learned to solve even before we reached quadratic equations in algebra? Now it was precisely a passage from this simple system of linear equations to a system with a countably infinite number of unknowns which gave to Volterra and Fredholm the basis of their theory of integral equations. At the limit the system of linear equations passes into a linear integral equation

$$
u(x)+\int_{a}^{b} u(\xi) K(x, \xi) d \xi=f(x)
$$

in which everything is given except the function $u(x)$ which is to be found. The solution $\left(x_{1}, x_{2}, \ldots, x_{n}\right)$ of the linear system with the indefinite increase of $n$ passes over into the solution $u(x)$ of the integral equation, but the legitimacy of the passage to the limit and of the solution must be verified in some manner. From the very outset the theory of integral equations has been found very useful in applications to mathematical physics and elsewhere. This might be expected because it is the generalization of the elemental theory of a set of linear equations.

The solution of integral equations is only one of many evidences of the mathematician's tightening grip upon the infinite. The infinite increase in the number of variables in our mathematical problems necessitates geometrically a theory of space of countably infinite dimensions. Already a promising beginning has been made by restricting the attention to Hilbertian space - that is, to that portion of infinitelydimensioned space for which $\sum x_{n}^{2}$, the sum of the squares of the coordinates of a point, is convergent. The familiar conditions for orthogonality of lines, etc., then generalize without material modification.

The theory of a space of infinitely many variables is companion to an incipient theory of functions of an infinite number of variables. I fancy a smile, inward or outward, on the faces of some of the physicists and astronomers present, to whom such a function theory may seem highly typical of the theoretic, up-in-theair character of the mathematician and his work. Let me try to address to you some arguments ad hominem. Have you nothing of such character in physics? In my early life, when I was hesitating between mathematics and physics as a profession, I first encountered Lagrange's generalized coordinates, whose number, by the way, need not be finite. The equations for motion, energy, etc., were set up in terms of these generalized coordinates. I shall never forget how the glittering generality of the theory astonished and fascinated me. And when the equations were actually applied to sundry objects - as, for instance, to the gyroscope - it seemed to me then, as it almost does still, about the finest example of getting something intellectually out of nothing that I had ever come across. The very generality of the coordinates gives to them adaptability to the varying complications of physical science. Now most phenomena of nature depend in their final analysis upon an 
infinite number of variables. How then can it be thought that we can master them unless the mathematician prepares the way by developing a theory of functions of infinitely many variables? The potential of force due to an electric current in a wire is a function of the shape of the wire and depends therefore upon the uncountably infinite number of its points. Indeed, it is not the pure mathematician who has been so hardy and rash as to attempt the beginning of a theory of functions of lines. No, the bold pioneer in this new field is no other than a mathematical physicist or, as we may claim with equa[1] truth, a physical mathematician-Volterra, a superb interpreter of current tendencies.

Time fails me to speak of the closely related and fundamental "functional calculus" initiated by Fréchet, certain aspects of which are being developed here at Chicago by Moore and his pupils.

An effect, and likewise a cause, of the generalized aims in mathematics, of which I have thus far spoken, has been the creation of refined mathematical tools. Under the term "mathematical tool" I mean to include concepts and algorisms which are usable for the demonstration of mathematical truth. The importance of concepts broadly comprehensive in their reach is shown alike by the history of geometry and of analysis. The open plane of Euclid yields a general geometry only after the introduction of ideal elements which we call the point, line, etc., at infinity. The creation thereby of a closed and perfect projective geometry was one of the milestones of mathematical progress in the century just past. Poncelet's comprehensive principle of geometric continuity, justified at first rather by necessity than by adequate logic, was another indispensable geometric creation. In the notion of a group we have a concept which reaches pervasively through the realms of geometry and analysis. Still another example of the fecundity of the subtler concepts forged by the progress of science is to be found in Hill's "periodic orbit," introduced in the theory of attraction as a norm from which to reckon the deviation of the orbit of the heavenly body.

My remaining illustrations will be taken chiefly from analysis, partly because it is the province with which I am most familiar, but also because the same general tendency which was exhibited conspicuously in the magnificent enlargement of geometry in the half century from 1820 to 1870 is to-day manifested in the broadening of analysis. Consider the familiar notion of an integral, apparently moulded to a finality in the hands of Riemann. This admits a finite number of discontinuities but an infinite number only under certain narrow restrictions. A totally discontinuous function - for example, one equal to zero in the rational points which are everywhere dense in the interval of integration, and equal to 1 in the irrational points which are likewise everywhere dense - is not integrable à la Riemann. The restriction became a very hampering one when mathematicians began to realize that the analytic world in which theorems are deducible does not consist merely of highly civilized and continuous functions. In 1902 Lebesgue with great penetration framed a new integral which is identical with the integral of Riemann when the latter is applicable but is immensely more comprehensive. It will, for instance, include the totally discontinuous function above mentioned. This new integral of Lebesgue is proving itself a wonderful tool. I might compare it to a modern Krupp gun, so easily does it penetrate barriers which before were impregnable.

Some of the newer mathematical concepts resemble the modern telescopes, disclosing new worlds of theorems and ideas bound together in a common system. Other concepts because of their high dispersive power act more like the optical 
grating, making new distinctions visible. This is particularly true of the modern theory of point sets which gives a grip on weird sets of points almost inconceivable in their irregularity. Take for consideration the two sets of points of which I spoke a few moments ago, the set of rational points everywhere dense and the set of irrational points likewise everywhere dense in a given interval, confusedly mixed together like grains of pepper and salt. Which predominates? Should the mixture be called white or black? Would it seem possible to say? Yet the theory of point sets tells us that the black - i.e., the irrational points - predominate; that the rational points are the exception and have a measure 0 , while the irrational points have a measure equal to the length of the interval. The rational points are weighed in the balance by Borel and Lebesgue and are found altogether lacking, at least in measure. They afford an example of what may be termed a null set, that is, a set of measure 0 . It is natural to expect that such a set of points would be without influence on many results - for example, on the value of a Lebesgue integral. This turns out to be the case. By allowing for a set of exceptional points of measure 0 many theorems can now be perceived and demonstrated which before were unthinkable.

Along with the widened aims and concepts of mathematics have come fresh methods and distinctive styles of thought. Look first at the older methods. The work of the grand old mathematicians of the seventeenth and eighteenth centuries was based on definite algorisms, such as the hypergeometric series of Gauss and the Taylor power series. Euler, in particular, was astonishingly prolific in algorisms. When the function theory sprang into existence it became even more indispensable to make elaborate studies of special algorisms and functions. A host of particular functions absorbed the attention of mathematicians - elliptic functions, automorphic functions, Bessel's functions, other functions defined by differential equations, and so on. The function theory of Weierstrass was based on a definite algorism, the Taylor power series. In recent times other processes and functions have received attention, for example, algebraic continued fractions and divergent series, factorial series, Dirichlet series, and so on. Definite algorismic work must, indeed, always remain of primary importance, not only on account of its use in calculation but also because the progress of our science continually introduces new functions for study. At the same time older processes need to be reexamined under modern lights, - for instance, the difference equation which has been considered by Nörlund, Birkhoff, and Carmichael in the light of the analytic function theory.

In sharp contrast with this algorismic work so characteristic of earlier time is a distinctively modern turn of thought which perhaps first found a clear exponent in Riemann. He presents a strange antithesis to his contemporary countryman, Weierstrass. Riemann bases the function theory upon a property rather than upon an algorism - to wit, the possession of a differential coefficient by the function in the complex plane. Thus at a stroke it is freed from dependence upon a particular process like the power series of Taylor. His celebrated memoir upon the $P$-function is a characteristic development of a whole Schar (family) of functions from their mutual relations.

A first aspect of the peculiar thought-mode of which Riemann's is a type is definition and development of a function from its properties. One illustration is found in the definition of a function by building a given region point for point and conformally upon another given region. In severe cases the regions may even contain an infinite number of leaves properly bound together. This illustration is significant 
also of a certain modern tendency to build mathematics upon correspondence and order. The boundary value problems of mathematical physics afford another example of definition of a function through its properties. As an effect of this tendency we may expect ultimately a great development of the incipient theory of functional equations. In this theory a single equation or system of equations expressing some property is taken as the definition of a class of functions whose characteristics, particular as well as collective, are to be developed as an outcome of the equations.

A second phase of the same thought tendency is to be found in the existence theorems so characteristic of modern analysis. Existence proofs are, indeed, a preliminary and indispensable element, if functions are to be defined through their properties. Their rôle has been discussed so often that I need not enlarge upon it.

A third phase of the same tendency is the modern examination of the postulates of geometry, arithmetic, algebra, and even mechanics. This postulational work seizes the material at the root before development sets in. It has to face the obvious objection that it is an exercise in sterile logic rather than creative mathematics. A swirling brain like Poincaré's will never stop in its constructive work for abstract postulational considerations, but will leap gaps like an electric spark. To this objection the rejoinder may be made that critical revision is not so far from creative thought as is generally supposed; that postulational theories are to-day much more fertile than in the past, that such postulational developments as Hilbert's in his famous Foundations of Geometry and his foundations for geometric group theory are, in fact, creative work of highest order; that Lebesgue formulated his concept of a generalized integral in the light of postulational requirements, and so on.

In this connection the primal thought which lies at the bottom of Moore's General Analysis deserves special consideration. When, he argues, a number of similar results in different fields are compared, the belief is forced irresistibly upon one that the essential oneness of the results must be due to identity of the hypotheses, this identity being partially or altogether veiled from sight by the admixture of foreign ingredients, as in chemical compounds. To apply a technical term, we have before us theories to be rendered simply isomorphic. The problem before the mathematician is accordingly to extract the common basis and exhibit the results as consequences of a common set of hypotheses applicable to varying conditions and objects. Thereby not only will like theories be coordinated, but the common underlying principles laid bare will be ready for use elsewhere. Doubtless the mathematician will continue to be guided for the most part by analogy in the future as in the past, but I doubt not that the isolation of the common elements in like theories will be stimulating and productive, even as the postulational study of the foundation of geometry has led to the discovery of new geometries. The general analysis has furthermore the advantage over ordinary postulational investigations of not tapping the root but the sap.

The change in method of which I have spoken centers largely in the prescription of properties and postulates rather than of algorisms. Entirely different from this is the increasing tendency to recast a problem so as to make it fertile. In the words which I have heard Hadamard employ, a problem must be "bien posé," fittingly formulated in order to give rich result. The inner nature of the problem needs to be comprehended. It carries in itself its own inherent limitations on its solution. To illustrate by drawing upon the calculus of variations, the problem of minimizing 
the integral

$$
\int F\left(x, y, y^{\prime}, y^{\prime \prime}, \ldots, y^{(n)}\right) d x
$$

by a function $f(x)$ is badly formulated and does not admit of precise answer unless we specify the class of functions from which the solution is to be taken. If we take, for example, the solution from functions of class $C^{(n)}$ in Bolza's terminology - that is, functions which are continuous together with their derivatives down to the $n$th inclusive - the problem then becomes well formulated and can be tackled successfully. In this particular case we impose limitations in order to well-formulate the problem. But it is distinctly modern to make a problem solvable by widening it so as to make it more instead of less comprehensive. Thus a problem not possible of general solution if only functions integrable in the ordinary Riemannian sense are considered, may become so if Lebesgue integrals are admitted. In fact, the solution may depend upon the kind of tool employed, and a careful study of the adaptation of the tool to the problem and of the problem to the tool should therefore be made. Thus if the problem should involve convergence, its character and formulation may change according as the tool involved is ordinary convergence, uniform convergence, quasi-uniform convergence, convergence "en moyen," or essentially uniform convergence.

In conclusion, partly by way of illustration and partly by way of summary, let me present a theorem which embodies most of the tendencies of which I have spoken. This is the so-called Fischer-Riesz theorem, remarkable for its elegant character. You all know the representation of a function $f(x)$ by a Fourier series,

$$
\frac{a_{0}}{2}+\frac{1}{\pi} \sum_{n=1}^{\infty}\left(a_{n} \cos n x+b_{n} \sin n x\right)
$$

in which the coefficients are obtained from the function by the equations

$$
a_{n}=\int_{-\pi}^{\pi} f(x) \cos n x d x, \quad b_{n}=\int_{-\pi}^{\pi} f(x) \sin n x d x .
$$

Now as Jacobi said, Man muss immer umkehren. Instead of representing a known function by a Fourier series, let us seek conversely to represent a given Fourier series by a function. The given series may be either convergent or divergent. We have now before us one of the great problems of analysis.

But before considering the problem let us obey the injunction: Man muss immer generalizieren. The Fourier series is only one of a class of series

$$
a_{1} \phi_{1}(x)+a_{2} \phi_{2}(x)+a_{3} \phi_{3}(x)+\cdots,
$$

in which the functions have with respect to a given interval $(a, b)$ the so-called orthogonality property,

$$
\int_{a}^{b} \phi_{i}(x) \phi_{j}(x) d x=0 \quad(i \neq j) .
$$

If, further, the functions have been normalized, as was the case above in the Fourier series, we have also

$$
\int_{a}^{b} \phi_{i}^{2}(x) d x=1
$$


Such a series is called a generalized Fourier's series, of which many special cases are well known, as, for instance, a series in terms of Legendre polynomials.

The problem now before us is to represent such a series, whether convergent or divergent, by a function and to derive the function from the series. We shall agree to say that a function $f(x)$ represents the series if 1 ) it is connected with the series by the equations

$$
a_{n}=\int_{a}^{b} \phi_{n}(x) f(x) d x
$$

and thus generates the series in the customary way; and if 2) the function agrees in value with the series at all points of the interval $(a, b)$ for which the series is convergent. The questions now confront us: When will such a function exist? How can it be obtained from the series? To what extent is it unique? In this shape the problem would be almost or quite insoluble. The problem is not yet "bien posé." For good formulation we must change the tool and use Lebesgue integrals throughout, thus extending the class of functions admissible for the representation of the series. Furthermore, in deriving the function from the series we will not require that the function and the series shall agree at all points of convergence but will allow exceptions at an infinite number of points forming a set of measure 0 .

It is now possible to state the theorem which Fischer and Riesz obtained independently by different methods. The necessary and sufficient condition that there shall exist a function representing the series (and having an integrable square $f^{2}(x)$ ) is that the sum of the square of the coefficients, $\Sigma a_{n}^{2}$, shall be convergent; in other words, the point $\left(a_{1}, a_{2}, \ldots\right)$ must lie within Hilbertian space of infinitely many dimensions. The function is completely determined save at an arbitrary set of points of measure zero, where we can give the function any value we choose. It can be obtained from the series by a proper grouping of the terms without change of order, which will render the series convergent except possibly at a set of points of measure 0 . The study of the convergence of the series has brought to light two new modes of convergence, one of which, Weyl's essentially uniform convergence, seems to me very important. The other I shall stop to explain briefly because it is an admirable example of generalized concept and because I think that it may interest those of you who are acquainted with the theory of least squares. If $S_{n}(x)$ denotes the sum of $n$ terms of our generalized Fourier series (I) and if $f(x)$ is, as before, the function represented by the series, the square of the error at any point $x$ of the interval is $\left[S_{n}(x)-f(x)\right]^{2}$, and the sum of the squares of the errors taken over the interval is the integral $\int_{a}^{b}\left[S_{n}(x)-f(x)\right]^{2} d x$. As $n$ increases indefinitely, this error integral approaches 0 , so that the series converges "en moyen" (on the average) although there may be no true convergence in the ordinary sense of the form. Conversely, if

$$
\int_{a}^{b}\left[S_{n+m}(x)-S_{n}(x)\right]^{2} d x
$$

can be made as small as we please by taking $n$ sufficiently great, then there exists a function $f(x)$ toward which the series converges en moyen.

But enough of this. I trust that you see that the Fischer-Riesz theorem affords an elegant example of modern mathematical research - characteristic in its generalized sweep, in its creation and use of refined tools and concepts, in its recasting of the problem so as to make it fertile, and lastly in its attainment of success through 
point set considerations, involving in this case the recognition of a null set of exceptional points. Not a little of the work of analysts for some time to come must center around the numberless new notions until their "Tragweite" - their carrying power and reach - is determined. To a great extent they have modified the character of current investigation. There is regretably less of the action and interaction of mathematics and mother nature. There is also insufficient study of approximation and astronomical mathematics - fields which are fortunately represented here by Moulton and MacMillan and which serve to counteract or supplement a somewhat widespread abstruse inclination in our country. But the newer thought does make tremendous advance when questions of theory rather than of calculation are involved, and the combination of sweeping generalization with rigor is astonishing. There remains yet to be accomplished the complete interweaving and correlating of the old and the new. We cannot see far into the future, but the well of mathematics will not run dry as long as there remains such springs as the problem of three or $n$ bodies, and the tantalizing, old, yet unsolved problem of the shape of curves of the $n$th degree.

UNIVERSITY OF WISCONSIN

JuNE, 1916 